\title{
Dissipative Particle Dynamics simulations of end-crosslinked nanogels
}

\author{
Veniero Lenzi ${ }^{\mathrm{a}, *}$, Marta M. D. Ramos ${ }^{\mathrm{a}}$, Luís S. A. Marques ${ }^{\mathrm{a}}$ \\ ${ }^{a}$ Center of Physics of Universities of Minho and Porto, University of Minho, Campus de \\ Gualtar, Braga 4710-057, Portugal
}

\begin{abstract}
Nanogels, due to their unique properties, have potential for promising applications in several fields. There is a growing interest in understanding which are the mechanisms controlling their properties, as those depend not only on composition, but also on internal structure. In this work Dissipative particle dynamics simulations were applied to simulate the formation of nanogels from monodisperse end-functionalized polymer chains, with the crosslinking reaction modelled using a distance-based algorithm, including a reversible intermediate step. Nanogel properties are studied by calculating their swelling transition curves and structure properties, such as network defects and entanglement state. Various chain lengths, reaction schemes, nanogel in silico synthesis methods and solvent ratios are compared and discussed, showing how these factors influence the nanogel swelling behaviour and structure. Our results show that, in order to be compared with experiments, nanogel simulations should carefully consider processing parameters.
\end{abstract}

Keywords:

Nanogels, Swelling, Entanglement, Dissipative particle dynamics, Network

*veniero.lenzi@fisica.uminho.pt 
formation.

\section{Introduction}

Micro- and nanogels, colloidal particles of 10-1000 nm in size [1], constitute nowadays a very important and fascinating class of macromolecules. According to their composition, microgels show a range of unique features, the most notable of which is the capacity to respond to external stimuli such as temperature [2], $\mathrm{pH}[3]$, salt concentration [4] and electric fields [5], amongst others. Moreover, nanogels are also very appealing from a fundamental point of view because of their dual colloidal and polymer nature [6], and they are currently investigated both theoretically [7, 8, 9] and experimentally $[10,11,12,13]$.

While poly(N-isopropylacrylamide) microgels are the most studied systems, due to their temperature controlled deswelling transition occurring at physiological ranges [1], polyurethane-based nanogels are particularly interesting biomaterials, due to their intrinsic versatility [14] and biocompatibility [15]. Indeed, they have been synthesized and applied in diverse biomedical applications, such as targeted drug delivery [16, 17, 18], vascular embolization [19] and to improve lifespan of shape memory biomaterials [20].

A novel method to synthesize PU-based hydrogels has been developed [21, 22 ], based upon the cyclotrimerization of isocyanate end-functionalized chains, which has been proven capable to provide networks with excellent swelling 
and improved mechanical properties, conjugated with a well-defined and controlled structure. To fully explore their potential, an investigation on how their structure and swelling properties may be controlled by different processing parameters is desired, since it is known the nano-scale features of a network are crucial in defining the material properties [23, 24].

While experimental studies are rapidly approaching the small time and length scales required to explore gel nanoparticles, simulations remain one of the most effective ways to investigate them [25, 26, 27, 28]. Regardless of the simulation method, the first and most important step is the in silico synthesis of the microgel particle itself. Many techniques have been devised for this, such as self-assembly of patchy colloidal particles [27], joining of randomly distributed crosslinks [29], chemical crosslinking reaction modelling [30]. Moreno and Lo Verso [30] demonstrated how the microgel internal structure could remarkably alter its kinetics, thus pointing out the importance of considering realistic structures. In this context, Dissipative Particle Dynamics (DPD) $[31,32,33,34]$ is emerging as a promising technique, because it reproduces the Flory-Rehner [35] theory in a natural way and allows for much larger time and length scales when compared with fully atomistic molecular dynamics. DPD has been confirmed capable to provide a good explicit representation of solvent and gel-solvent interactions [28, 29], thereby representing an appropriate choice to study nanogels within a solvated environment. As a matter of fact, DPD was used to study nanogels with an inner diamond-like network $[36,37,38]$ or structures obtained by joining randomly distributed crosslinks [29].

In this work, we simulated the formation of crosslinked nanogels by the 
reaction of end-functionalized chains, and to investigate their properties in terms of swelling transition curves, structure factors and density profiles, average elastic strand and minimal loop lengths. A two-step reversible reaction scheme has been implemented within the framework of DPD simulations, based upon the isocyanate trimerization process, and it has been compared with a more traditional crosslinking reaction. Furthermore, two different in silico protocols to obtain the nanogel from network were compared, as well as various solvation states during the network assembly phase.

\section{Methods}

\subsection{DPD simulation parameters and system preparation}

Throughout this work, reduced DPD units will always be used, with $\tau$ denoting the DPD time unit. In dissipative particle dynamics simulations, DPD particles are generally coarse-grained representations of one -or moremonomer. Any DPD particle $i$ is subject to the DPD force $f_{i}$, composed by a pair term $F_{C}$, a dissipative term $F_{D}$ and a random force term $F_{R}$ :

$$
\begin{array}{r}
\vec{f}_{i}=\sum_{j}\left(\vec{F}_{C, i j}+\vec{F}_{D, i j}+\vec{F}_{R, i j}\right) \\
\vec{F}_{C, i j}=a_{i j} w(r) \hat{r}_{i j} \\
\vec{F}_{D, i j}=-\gamma w^{2}(r)\left(\hat{r}_{i j} \cdot \vec{v}_{i j}\right) \hat{r}_{i j} \\
\vec{F}_{R, i j}=\sigma w(r) \theta_{i j}(t) \hat{r}_{i j} \\
w(r)=1-\frac{r}{r_{c}},
\end{array}
$$

where $r_{c}$ is the cut-off radius of DPD interactions, set to $r_{c}=1$. The

function $\theta_{i j}(t)$ is a randomly fluctuating variable $[32,33]$, the damping factor 
$\gamma$ is set equal to 4.5 and $\sigma^{2}=2 k T \gamma$. The density of all systems was set to $\rho=3$, while the temperature $T=1$ was used for all simulations. The timestep is $\Delta t=0.02$.

The pair repulsion parameters $a_{i j}$ are connected to the Flory-Huggins parameters $\chi_{i j}$ by eqn. (6) [33], which in turn may be related to the Hildebrand solubility parameters, eqn. (7) [39]:

$$
\begin{array}{r}
a_{i j}=a_{i i}+3.27 \chi_{i j} \\
\chi_{i j}=\frac{v_{i j}}{R T}\left(\delta_{i}-\delta_{j}\right)^{2} \\
\delta_{i}=\left(\frac{\Delta H_{v a p}-R T}{v_{i}}\right)^{0.5}
\end{array}
$$

where $\Delta H_{v a p}$ is the enthalpy of vaporization, and $v_{i}, v_{i j}$ are the molar and partial molar volume, respectively. Equations (6) - (8) therefore suggest a bottom-up approach to obtain the $a_{i j}$ from atomistic simulations, as done in $[40]$.

Liquid state Molecular Dynamics simulations were performed to obtain the $\Delta H_{v a p}$ and $\delta_{i}$ of every different gel constituent, by considering a PEGPU isocyanate-end crosslinked nanogel as a model system. The GAFF-IC force field [41], an optimized version for isocyanates of GAFF [42] was used. The interaction parameters are reported in table 1 for the different types of beads. To calculate $\chi_{i j}$ an unique average bead volume of $v \equiv 110 \AA^{3}$ was used. Incidentally, this also defines the length scale of the simulation: $r_{D P D}=(\rho v)^{\frac{1}{3}}=6.91 \AA$. The repulsion parameters $a_{i j}$ are equal to 25 when $i=j$.

Concerning solvent-gel interactions, the solvent has been made insensitive of polymer bead type and only one repulsion parameter $a_{s g}$ was used to treat 
Table 1: DPD interaction parameters for all species appearing in the simulations. Ring indicates an isocyanurate ring, that is a fully crosslinked bead. Concerning the not fully crosslinked beads, their DPD parameters are assumed to be equal to those of isocyanate beads.

\begin{tabular}{l|ccccc} 
Bead type & PEG & Urethane & Isocyanate & Ring & Alkyl \\
\hline PEG & 25 & 25.69 & 25.03 & 26.32 & 36.02 \\
Urethane & & 25 & 25.42 & 25.10 & 31.9 \\
Isocyanate & & & 25 & 25.93 & 34.85 \\
Ring & & & & 25 & 29.70 \\
Alkyl & & & & & 25
\end{tabular}

all the interactions between nanogel and solvent. To replicate the swelling transition, $a_{s g}$ was varied from 15 (very good solvent) to 50 (very poor). Good solvent condition will always refer to $a_{s g}=25$.

Polymer chains were represented using a bead-spring model, with harmonic bond and bending potentials defined as:

$$
\begin{gathered}
V_{\text {bond }}=K_{\text {bond }}\left(r-r_{0}\right)^{2} \\
V_{\text {angle }}=K_{\text {bend }}\left(\theta-\theta_{0}\right)^{2} .
\end{gathered}
$$

In particular, the bending potentials allow to better reproduce the stiffness of the polymer chains $[43,44]$. Regardless their type, all DPD beads are assumed to have the same mass and volume. The equilibrium length of all bonds was set to 0.434 , corresponding to the average center-of-mass distance of PEG monomers in the atomistic representation, and a stiff spring constant $K_{\text {bond }}=$ 500 was used. Such choice helps to prevent unphysical chain crossings. To 
further enforce this condition, a segmental repulsive (SRP) potential [45] was used, with a range of 0.4 and a force constant of 100. Equilibrium angles of $\pi / 2$ radians and a spring constant $K_{\text {bend }}=0.5$ were adopted, except when the vertex is a crosslinking (CL) bead, in which case $\theta_{e q}=2 \pi / 3$ radians.

Initial polymer and polymer-solvent configurations were randomly generated in a cubic periodic box at the target density, and equilibrated for 50000 $\tau$. Three independent runs were considered for every system. All simulations were performed using LAMMPS [46] and the DPD implementation therein. For each system considered, three statistically independent runs were performed and their results averaged.

\subsection{Crosslinking reaction modelling}

The isocyanate end-crosslinking reaction might be seen as a two-step reaction (see figure 1), in which an activated dimer is formed first and a stable trimer is obtained when another isocyanate comes nearby and reacts. To model this, a distance-based routine has been adopted [47, 48], which searches for all the reactive beads closer than $d=0.8$. Each reaction has a predetermined probability to happen, under the constraint that any bead cannot react more than once per iteration. To take into account reversibility, bond-breaking reactions were also included. Equal probabilities $p=0.4$ were set for dimerization and trimerization, while a $p=0.04$ was chosen for the activated dimer dissociation. Reactive iterations are performed every $2.5 \tau$,

to guarantee proper relaxation and diffusion between reaction steps. Newly created bonds were directly added with their equilibrium lenthghs and force constants. Despite this, no abrupt changes in potential energy or temperature were observed. 
a)

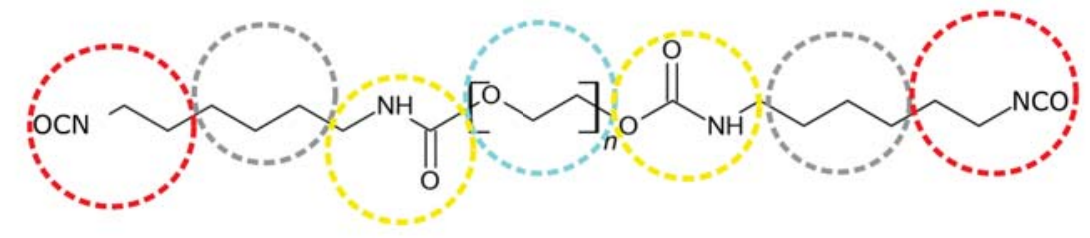

Polymer bead

b) Part. reacted crosslinker

Fully reacted crosslinker

Alkyl bead

Urethane bead

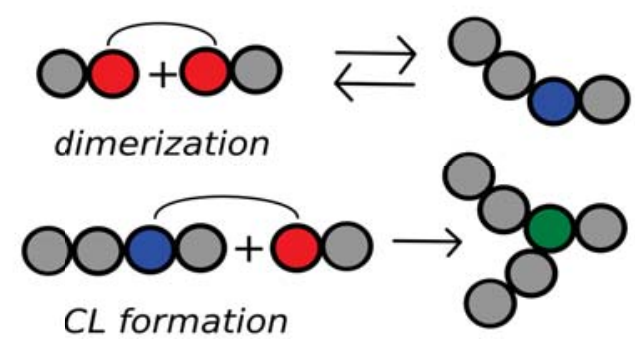

Precursor melt

c)

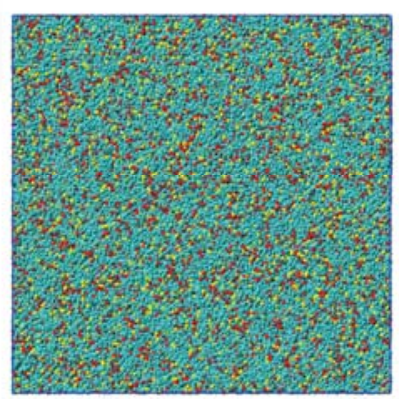

Network

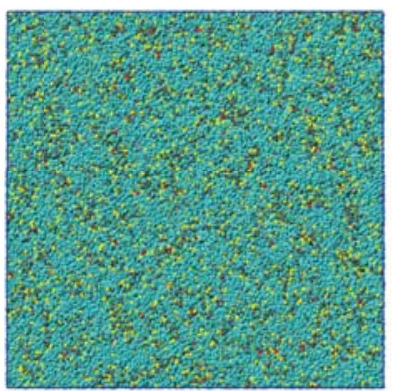

Nanogel

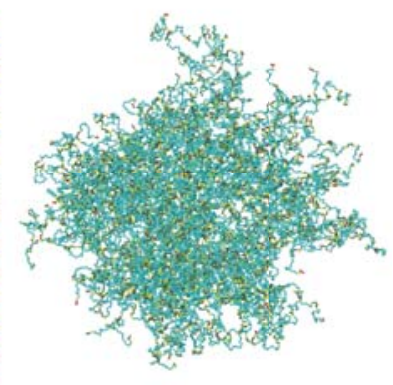

Figure 1: a) The structure of a precursor isocyanate-end functionalized polymer chain.

The circles identify the corresponding DPD beads.b) The crosslinking reaction scheme, modelled as a two-step process.c) Representations of the precursor melt(left), the reacted network(center) and the obtained nanogel(right) for a $n=20$ system. In the nanogel picture, solvent is not shown. 
The formation process was carried on until at least $90 \%$ of the initial reactive bead content is fully reacted. To achieve this, 5000 to 40000 iterations were required, depending upon the system under consideration (see supplementary information for more details). For the interested reader, source code for the reaction routine is available upon request.

\subsection{Nanogel bead construction, swelling and characterization}

Nanogel beads were cut from the crosslinked network, in such a way that all chains severed in correspondence of internal bonds were entirely stripped off. The resulting disconnected parts were also removed. To avoid bonds crossing the periodic boundaries, the maximum allowed cut-off radius is $r_{\max }=L / 2-0.5, L$ being the periodic box length. At the same time, the cut-off radius should be large enough to ensure a good representation of the internal structure of the nanogel and reduce size effects. In this sense, a minimum cut-off radius of 10.5 was adopted (further details on the determination of this quantity are reported in supplementary information).

Alternatively, the reaction can be performed in spherical confinement region, so that the final reaction product is already a nanogel and no further

handling of the system is needed. A third approach [29] consists in joining a set of randomly distributed CL beads with chains of the desired length, under periodic boundary conditions. In this case the cut still needs to be done, but the reaction phase is completely skipped. It is worth nothing that, unlike the others, this method does not contemplate any equilibration phase, since the initial CL beads' positions are generated randomly.

Subsequently, nanogels were then put into a larger periodic box, with at least $L=50$ and solvent particles were added in the required amount to have 
$\rho=3$. The centre of mass of the nanogel was kept fixed to the centre of the box. Solvated systems were equilibrated under good solvent conditions for at least $10000 \tau$. The gyration radius $R_{g}$ was adopted as an estimation of the nanogel size. The swelling ratio is here defined as the ratio between $R_{g}\left(a_{s g}\right)$ and the collapsed state $R_{g, c}$, defined as $R_{g, c}=R_{g}(50)$.

The form factor $P(q)$ has been calculated as:

$$
P(q)=\frac{1}{N} \sum_{i, j=1}^{n} e^{-i\left\langle\mathbf{q}\left(\mathbf{r}_{i}-\mathbf{r}_{j}\right)\right\rangle},
$$

where the sum runs over all the nanogel beads and angle brackets indicate an average over the possible directions of $\mathbf{q}$ and over time.

The swelling curves were obtained by changing the value of $a_{s g}$. For each value, simulations $6000 \tau$ long were run, the last $2000 \tau$ of which being used for data production.

The network topology has been inspected by counting CL and linear linkage (LL) beads, as well as CL beads concentration and CL functionality, that is the average number of bonds involving both CL and LL beads. Furthermore, network defects such as dandling chains and single-chain loops were counted, along with loops made up by two chains. To gain information upon the entanglement state of the system, a primitive path analysis (PPA) [49] was performed using the Z1 code [50, 51, 52, 53].

\section{Results and Discussion}

\subsection{Chain length dependence}

Nanogels were obtained from reacted networks made from $n=10,20$ and 40 chains by carving them out from the melt. 
As shown in figure 2, all nanogels undergo the expected swelling transition when put in solvent. In the collapsed state $\left(a_{s g}=50\right)$, they assume a spherical shape with well-defined borders and a central density of 3.5, regardless of the internal structure. This high density value is caused by the short equilibrium bond lengths adopted in this model. By using the typical value of $0.6[29]$, a density of 3 is recovered. In terms of swelling ratios, the results are unaffected by this change, as setting larger bonds is equivalent to a rescaling of the bead internal distances. So, it was decided to present here the results with the shorter bond lengths, as they were obtained through a mapping of an all-atom representation to DPD.

On the other hand, nanogels can be clearly differentiated when swollen. As the precursor chain length is increased, lower CL beads densities are found, resulting in an increase of the average elastic strand length, as can be seen in table 2. Consequently, longer chain-made nanogels present larger radii and lower densities in the swollen state. The calculated structure factors confirm these observations, showing the hard-sphere scattering pattern for a collapsed nanogel and fuzzy sphere curves in the swollen state. Regarding the latter, is possible to see that the first $P(q)$ peak, corresponding to length scales typical of the nanogel sizes, are sharper for shorter precursor length nanogels. Finally, it is worth noting that a sharper swelling transition is found for longer chain networks, as predicted by theory [35].

Concerning their topological features, the lower CL functionality found in longer chain networks reflects in an increase in the percentage of elastically inactive defects, which includes both dandling ends and tadpoles. Interestingly, the increased number of defects for $n=40$ is mainly due to a larger 

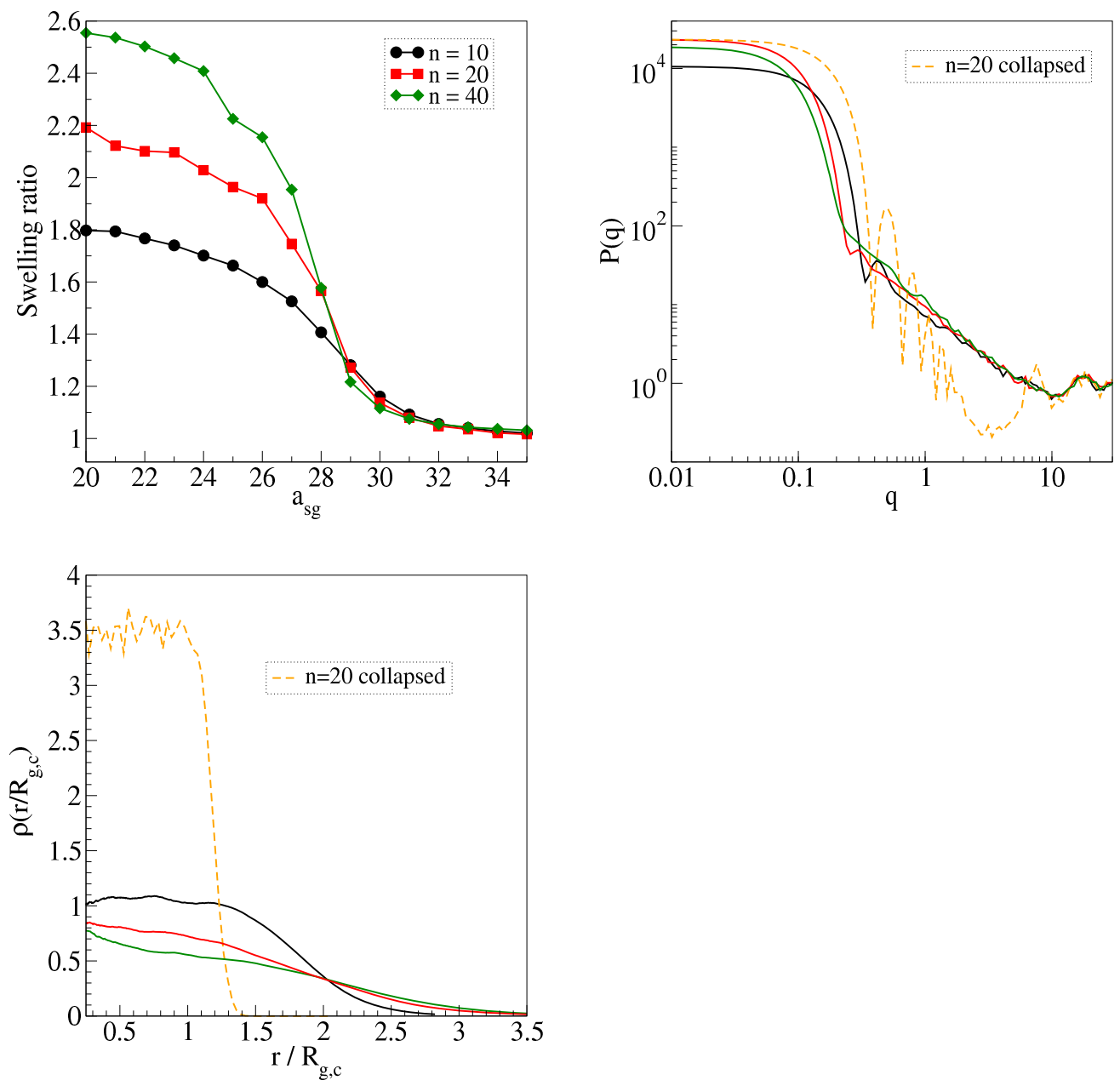

Figure 2: Swelling transition curves(top left), structure factors (top right) and radial density profiles in good solvent(bottom) of $n=10,20$ and 40 end-crosslinked nanogel beads. The form factor and radial density of the $n=20$ nanogel in the collapsed state are also shown as a dashed line. 
Table 2: Topological characterization polymer networks obtained for different initial chain lengths. The elastically inactive defects (1-defects) and 2-loops are expressed as a percent ratio with respect to the initial chains. $\langle N\rangle$ is the number of beads per strand and $\langle Z\rangle$ is the number of kinks per chain. Numbers in brackets represent the uncertainty.

\begin{tabular}{lcccccc} 
System & CL density & CL functionality & 1-defects & 2-loops & $\langle N\rangle$ & $\langle Z\rangle$ \\
\hline 10 & $5.30(0.01) \%$ & $2.77(0.01)$ & $9.17(0.54) \%$ & $1.36(0.06) \%$ & $11.33(0.01)$ & $0.025(0.001)$ \\
20 & $3.02(0.01) \%$ & $2.71(0.01)$ & $13.02(0.27) \%$ & $1.07(0.32) \%$ & $21.36(0.02)$ & $0.153(0.01)$ \\
40 & $1.14(0.01) \%$ & $2.63(0.01)$ & $18.38(1.02) \%$ & $0.81(0.35) \%$ & $41.39(0.07)$ & $0.47(0.01)$
\end{tabular}

number of dandling chains, while the number of tadpoles is significantly lower. This is a consequence of the fact that the probability for two ends of the same chain to find themselves in the same point is reduced as the chain length increases. In what concerns the entanglements, the network formation freezes the pre-existent state and changes the melt entanglement state only slightly.

\subsection{Comparison of in silico nanogel synthesis techniques}

Since there are several ways to perform the in silico synthesis of the nanogel, it is important to consider how such a choice could alter the calculated quantities of the simulated system. In figure 3 the swelling curves, structure factors, radial density and means squared internal bead distances (MSID) profiles are reported for three possible ways to obtain nanogel beads from a $\mathrm{n}=20$ precursor melt:

- (random-made) $\mathrm{Cl}$ points are randomly joined with linear chains,

- (confinement-made) or by letting the melt self-assemble in a confined region, 
- (cut) letting the melt self-assemble under periodic conditions, and cut the nanogel afterwards.

Table 3: Topological characterization of networks made from a $n=20$ chain melt, using different in silico methods. The elastically inactive defects (1-defects) and 2-loops are expressed as a percent ratio with respect to the initial chains. $\langle N\rangle$ is the number of beads per strand and $\langle Z\rangle$ is the number of kinks per chain. The first row, already appeared in table 2 , is reported here for comparison. Numbers in brackets represent the uncertainty.

\begin{tabular}{lcccccc} 
System & CL density & CL functionality & 1-Defects & 2-loops & $\langle N\rangle$ & $\langle Z\rangle$ \\
\hline 20 cut & $3.02(0.01) \%$ & $2.71(0.01)$ & $13.02(0.27) \%$ & $1.07(0.32) \%$ & $21.36(0.02)$ & $0.153(0.01)$ \\
20 confined & $3.08(0.01) \%$ & $3.00(0.01)$ & $20.93(2.26) \%$ & $1.40(0.17) \%$ & $21.35(0.01)$ & $0.10(0.01)$ \\
20 random & $1.73(0.01) \%$ & $2.12(0.01)$ & $1.025(0.04) \%$ & $1.73(0.12) \%$ & $21.08(0.01)$ & $0.65(0.07)$
\end{tabular}

The random-made nanogels show the lowest swelling ratio and the highest density in the swollen state, with a significant deviation with respect to the other simulation designs. Long simulations were performed to verify whether the difference in swelling ratios could be caused by the randommade nanogels not being fully relaxed. However, it was found that, in good solvent conditions, the nanogel radius is not changing over periods of at least 200 swelling relaxation times(more details can be found in supplementary information).

By contrast, both of the reaction based nanogels allow for larger swollen sizes, the cut one being the largest, and practically equal densities for $r / R_{g, c}<$ 1.5. The main difference between the two is actually the skin layer, which is wider and less defined in the cut nanogel. The form factor reflects these differences, with a more compact structure found for the random-made nano- 

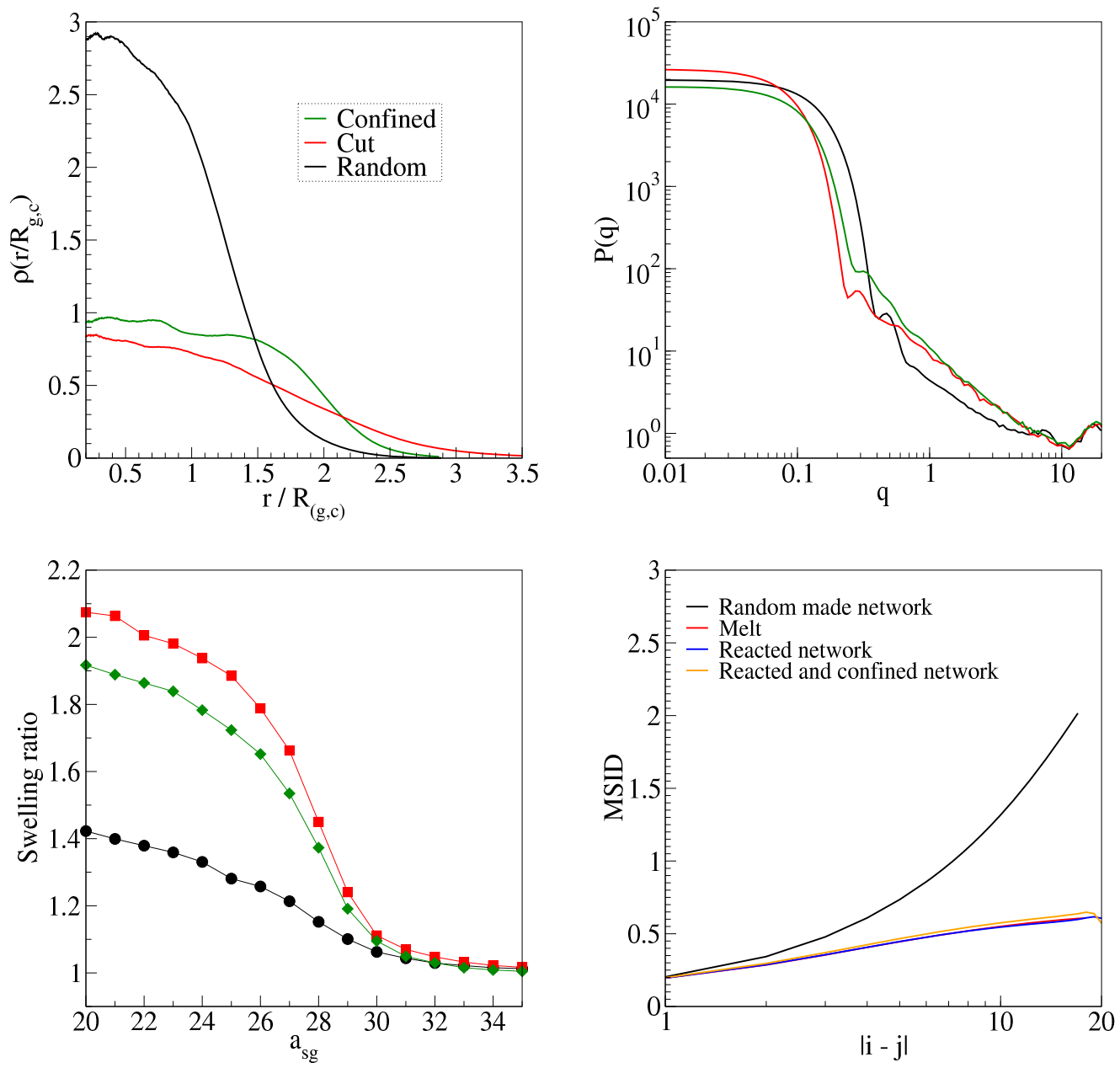

Figure 3: Radial density profiles (top left), form factors (top right), swelling transition curves (bottom left) and mean squared internal distances (bottom right) of $n=20$ nanogel beads obtained with three different in silico methods. 
gel, with the first peak more visible and shifted at larger $q$, while the other beads show qualitatively the same $P(q)$.

From table 3, it can be observed that the nanogel made under confinement has a higher CL density and functionality. It is then natural to expect, as observed, a lower swelling ratio. In what concerns topology, the confinementmade network has a lower value of $\langle Z\rangle$ when compared to periodic networks, which is a surface effect. At the same time, the number of elastically inactive defects is slightly larger.

Concerning the random-made network, a much lower CL density and functionality is found, although the number of elastically inactive defects is sensibly lower and the higher order loops are virtually inexistent. On the other hand, a much larger $\langle Z\rangle$ is found for them, indicating that randommade networks are much more entangled than the others. This seems contradictory, since a poor CL density suggests larger swelling ratios, while greater $\langle Z\rangle$ suggests the opposite. However, there is also another aspect that should be considered. Due to the formation process, which directly provides the crosslinked network without any previous equilibration, the random-made network is mainly constituted by stretched chains subject to an entropic elastic force which acts to contract them, thereby opposing and hindering any solvent-induced swelling. This can be easily revealed by calculating the mean squared internal distance $[54,49]$ for the unreacted $\mathrm{N}=20$ melt and the different networks. While the reaction-based network formation results in a final structure with chains still having a internal distance distribution very close to that of the precursor melt, for the random-made network MSID shows a significant deviation, meaning that the chains are overstretched. 


\subsection{Comparison of reaction routes}

Apart from end-crosslinking, we considered a more traditional crosslinking technique, in which the end-reactive chains covalently bond to polyfunctional CL small molecules, as sketched in the top left panel of figure 4. This way, we could assess the differences of nanogels simulated using both reaction schemes. Stoichiometric mixtures of trifunctional crosslinkers were mixed to $n=16$ precursor chains, the ends of which could react with a probability coefficient of 0.4 with the functional CL beads. In this way, network strands would have the same length as for the end-crosslinking case. Two different crosslinker formulations were used: one fully made of trifunctional crosslinker and a more realistic one, containing a $30 \%$ weight ratio of 4-functional impurities. This reflects the fact that a certain degrees of higher functionality impurities is always present in commercial isocyanurate products. Since only a one-step process is considered this time, the reaction speed is significantly faster, and fully formed network can be reached in only 5000 iterations.

All nanogel beads were cut from a periodic network of $n=20$ chains and contained roughly 10000 beads. During equilibration and network formation, the mixtures always appeared well mixed and no phase separation was observed.

Figure 4 reports the form factors, swelling ratios and radial density profiles at $a_{s g}=25$, for polymer networks obtained in three different ways, the properties of which are summarized in table 4. For the impure mixture, the stoichiometric ratio between chain and crosslinker ends is not exactly matched, hence lower products are expected. 


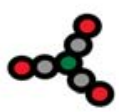

3-functional CL

4-functional impurity

- $-\mathrm{H}$ termination
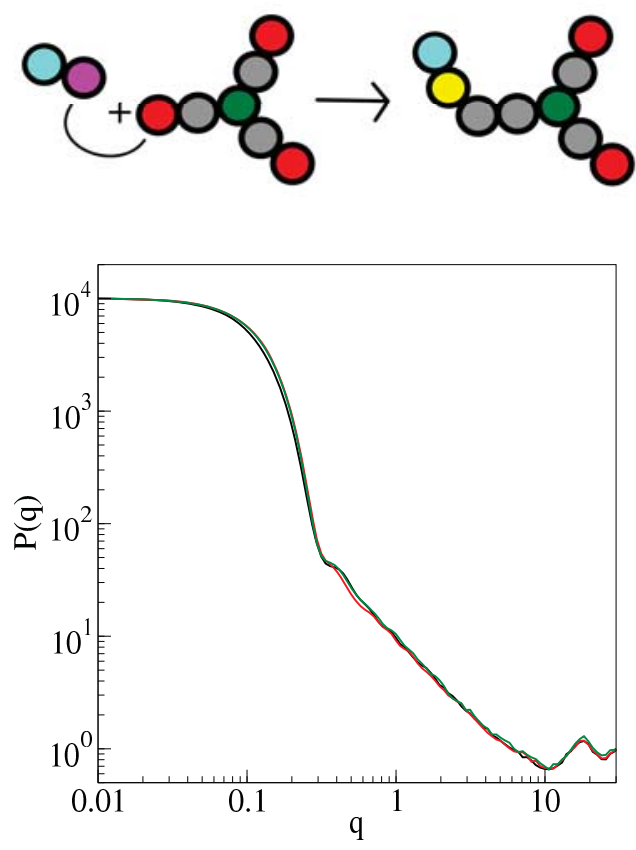
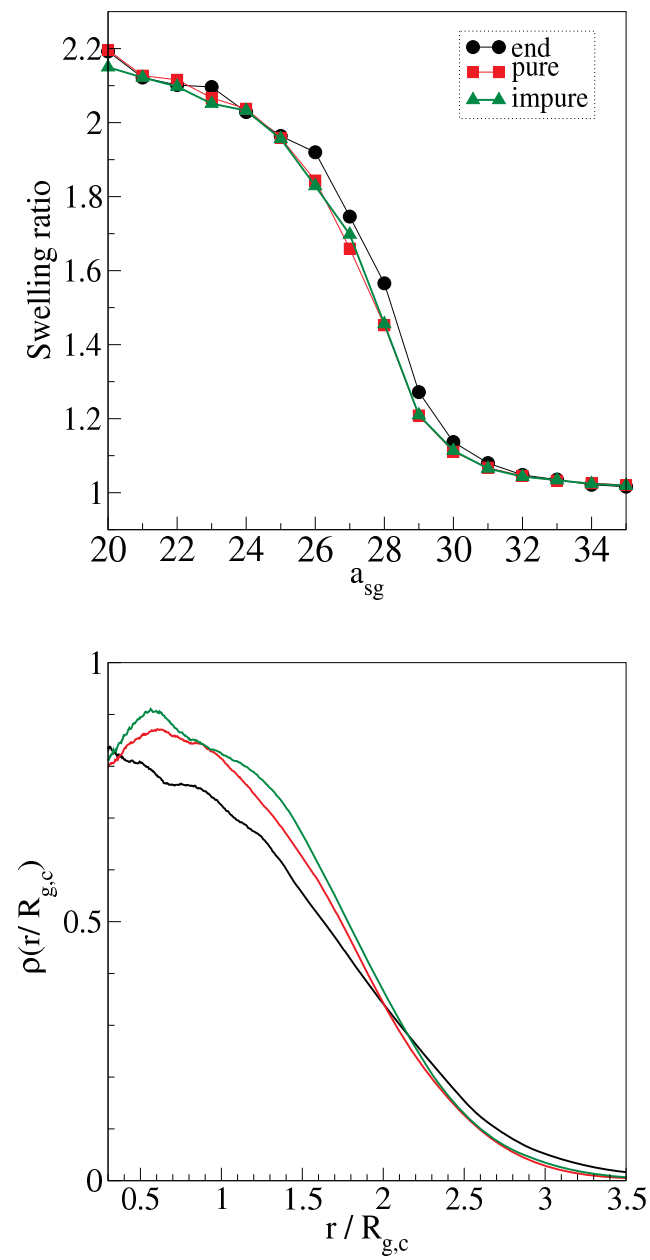

Figure 4: The structure of the crosslinking molecules and schematics of the alternative reaction scheme are in the top left panel. The colour legend is the same as in figure 1. Swelling curves (top right), form factors (bottom left) and radial density profiles (bottom right) in good solvent conditions are also reported for the nanogels obtained considering different chemical routes and crosslinker purity level. 
The structure factor is not revealing significant differences between the differently processed nanogels. However, the end-functionalized ones exhibit slightly larger swelling ratios and lower radial densities, whereas pure and impure chain-crosslinker made nanogels exhibit very similar swelling ratios. Therefore, the two synthesis methods provide negligible differences in terms of swelling properties and also in averaged strand and loop lengths. The enhanced swelling of the end-crosslinked nanogels is related to the lower overall content of crosslinking beads.

Interestingly, the presence of impurities in the crosslinker composition does not seem to impact significantly the swelling ratios. This is expected as the lower number of bonds due to the stoichiometric impairment is compensated by the presence of effective 4-functional CL beads, tightening the structure, as reflected by the smaller average chain length $\langle N\rangle$. In terms of defects, it is interesting to observe that the mixing approach significantly reduced their occurrence. The number of kinks per chain is also larger for the system containing impurities.

Table 4: Comparison of topological properties of $n=20$ networks obtained with different chemical routes. The elastically inactive defects(1-defects) and 2-loops are expressed as a percent ratio with respect to the initial chains. $\langle N\rangle$ is the number of beads per strand and $\langle Z\rangle$ is the number of kinks per chain. The first row, already seen in table 2, is reported here for comparison. Numbers in brackets represent the uncertainty.

\begin{tabular}{lcccccc} 
System & CL density & CL functionality & 1-defects & 2-loops & $\langle N\rangle$ & $\langle Z\rangle$ \\
\hline end-crosslinked & $3.02(0.01) \%$ & $2.71(0.01)$ & $13.02(0.27) \%$ & $1.07(0.32) \%$ & $21.36(0.02)$ & $0.153(0.01)$ \\
pure-mix & $3.53(0.01) \%$ & 3 & $7.58(0.85) \%$ & $1.10(0.13) \%$ & $21.51(0.06)$ & $0.173(0.03)$ \\
impure-mix & $3.72(0.01) \%$ & 3 & $12.85(0.54) \%$ & $1.25(0.20) \%$ & $19.97(0.01)$ & $0.170(0.02)$
\end{tabular}


This comparison is useful to assess the effectiveness of an end-crosslinking reaction. Since no phase separation was observed in the 2-component precursor melts, their final network structures are very close to an ideal realization, especially when pure trifunctional crosslinkers are employed. Therefore, having found very similar results with end-crosslinked structures confirms that very well-structured networks can be easily obtained through this technique. This represents a clear advantage because this process, apart form catalyst and solvent, involves only one component.

\subsection{Effect of explicit solvent inclusion}

In real systems, crosslinking processes commonly take place in solvated environments, hence it is important to consider how explicit solvent inclusion during the network formation process can influence the computational predictions. Different solvation levels, from 0 to $66 \%$, were considered during the network assembly of $n=10$ chains, from which nanogels containing about 13000 beads were cut. It is expected that the end-crosslinking reaction would be slower due to reduced reactant concentration. This is indeed the case, as it can be observed in figure 5, where the CL bead formation speed decreases as the solvent concentration increases.

In addition, the explicit solvent inclusion produces a sensible increase of the nanogel swelling in good solvent even though, as shown in table 5, the CL concentrations and functionality are very similar for all the solvent percentages. This increased swelling ratio is due to two key factors. First, as the solvent percentage increases, so does the incidence of elastically inactive defects and 2-loops. This can be understood considering that the solvent presence decreases the overall chain density, thereby reducing the probability 
Table 5: Topological characterization of networks made from a $n=10$ chain melt, under the presence of solvent at different weight ratios during their formation. The elastically inactive defects (1-loops) and 2-loops are expressed as a percent ratio with respect to the initial chains. $\langle N\rangle$ is the number of beads per strand and $\langle Z\rangle$ is the number of kinks per chain. Numbers in brackets represent the uncertainty.

\begin{tabular}{lcccccc} 
Solvent $\%$ & CL density & CL functionality & 1-defects & 2-loops & $\langle N\rangle$ & $\langle Z\rangle$ \\
\hline 0 & $5.33(0.01) \%$ & $2.78(0.01) \%$ & $9.17(0.54) \%$ & $1.36(0.06) \%$ & $11.33(0.01)$ & $0.025(0.001)$ \\
33 & $5.66(0.01) \%$ & $2.84(0.01) \%$ & $16.33(0.21) \%$ & $1.89(0.47) \%$ & $11.31(0.01)$ & $0.011(0.002)$ \\
50 & $5.60(0.01) \%$ & $2.86(0.01) \%$ & $18.86(1.28) \%$ & $2.22(0.17) \%$ & $11.30(0.01)$ & $0.004(0.002)$ \\
60 & $5.64(0.01) \%$ & $2.86(0.01) \%$ & $21.34(0.56) \%$ & $2.72(0.21) \%$ & $11.27(0.01)$ & $0.003(0.001)$ \\
66 & $5.77(0.01) \%$ & $2.87(0.01) \%$ & $24.43(1.53) \%$ & $3.23(0.21) \%$ & $11.27(0.01)$ & $0.001(0.001)$
\end{tabular}

of two chain ends from different chains to come in close contact and react.

Second, the number of kinks per chain is decreasing as solvent is added. Clearly, this effect is expected to be more significant for longer chains, as the $\mathrm{n}=10$ presented here are almost unentangled.

The addition of solvent has thus a strong effect on the simulated networks, dramatically changing their topology and, by consequence, their macroscopic properties.

\section{Conclusions}

In this work, the assembly of isocyanurate-crosslinked networks from endfunctionalized chains has been investigated by using a bead-spring polymer model in conjunction with a scheme for the chemical crosslinking reaction and dissipative particle dynamics simulation with MD-derived parameters.

Realistic nanogels with the typical core-corona structure and the swelling 

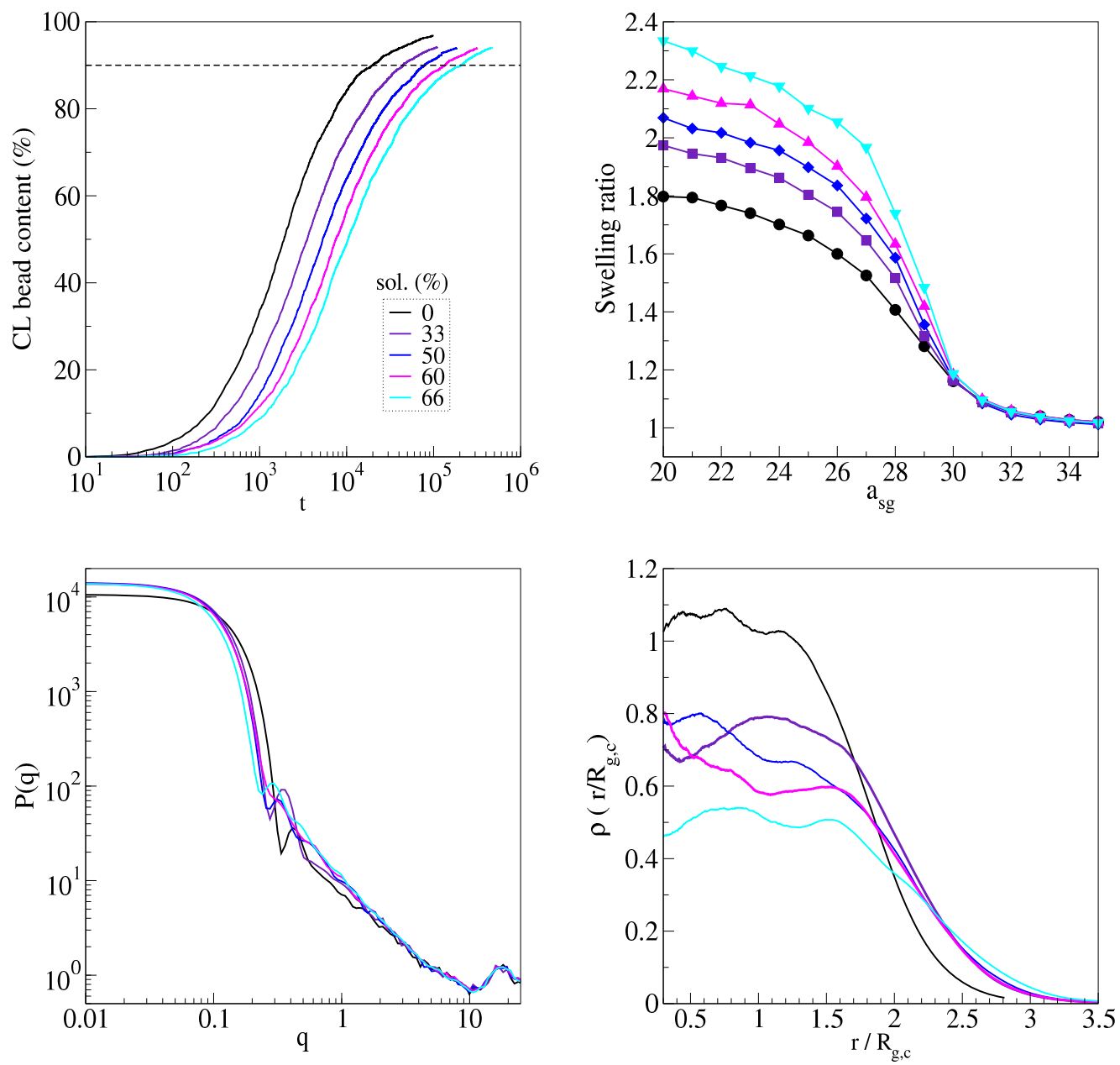

Figure 5: Reaction rates(top left), swelling curves (top right), form factors (bottom left) and radial density profiles (bottom right) in good solvent conditions of $\mathrm{n}=10$ nanogels obtained at different solvation ratios during the network formation phase. 
transition were obtained, equivalent to those made from ideal prepolymercrosslinker mixtures. It is worth noting that the techniques employed can be used as well for any isocyanurate-crosslinked material, and the reaction scheme can be easily modified to take into account more complex chemical networks.

Concerning the nanogel properties, it was shown that their swelling ratios are strongly dependent not only the precursor features, such as chain length, but also on the processing route -or simulation design- chosen. Indeed, from the same precursor, it was possible to make nanogels with a dramatically different swelling behaviour, demonstrating that the in silico techniques tested here are inequivalent, and the choice upon which one to employ should be made in an informed way. Moreover, apart from the swelling differences, the networks also look structurally different, with different degrees of entanglement and concentration of defects.

Nanogel swelling ratios and, by extension, network elastic properties are indeed intimately connected with topological features, in particular with chain entanglement and elastically inactive defects, as shown through their inspection and primitive path analysis. As it is made clear in the solvation study, these two factors are both affecting the final network properties, with less-entangled and more defective networks obtained as the solvent content is increased during the crosslinking phase.

The model can be improved in several ways, for instance by introducing extra terms for specific interactions such as those involving urethane $[55,56]$ and isocyanurate[57] groups, but it is remarkable that it is already capable to capture most of the relevant features of end-crosslinked networks. 


\section{Acknowledgements}

The authors wish to thank Dr. Dirk J. Dijkstra and Dr. Piet J. Driest for the fruitful discussions and ideas, and Prof. Martin Kröger for kindly providing the Z1 code for primitive path analysis. This project has received funding from the European Union's Horizon 2020-Research and Innovation Framework Programme under the Marie Skłodowska-Curie Grant Agreement no. 642890 (http://thelink-project.eu/) and it was partially supported by the Portuguese Foundation for Science and Technology (FCT) in the framework of the Strategic Funding UIDB/04650/2020-2023, by the FCT grant SFRH/BD/128666/2017. The authors acknowledge FCT and the National Advanced Computing Network (RNCA) for providing HPC resources from cluster Bob at Minho Advanced Computing Center (MACC). Such resources have contributed to the research results reported within this paper. URL: https://www.fccn.pt/computacao/rnca and https://macc.fccn.pt

\section{References}

[1] A. Fernandez-Nieves, H. M. Wyss, J. Mattsson, D. A. Weitz (Eds.), Microgel Suspensions: Fundamentals and Applications, Wiley-VCH, Weinheim, Germany, 2011.

[2] R. Pelton, Temperature-sensitive aqueous microgels, Advances in Colloid and Interface Science 85 (1) (2000) 1 - 33 . doi:https://doi.org/10.1016/S0001-8686(99)00023-8.

URL http://www.sciencedirect.com/science/article/pii/S0001868699000238 
[3] L. He, D. E. Fullenkamp, J. G. Rivera, P. B. Messersmith, ph responsive self-healing hydrogels formed by boronate-catechol complexation, Chem. Commun. 47 (2011) 7497-7499. doi:10.1039/C1CC11928A.

URL http://dx.doi.org/10.1039/C1CC11928A

[4] T. López-León, A. Fernández-Nieves, Macroscopically probing the entropic influence of ions: Deswelling neutral microgels with salt, Phys. Rev. E 75 (2007) 011801. doi:10.1103/PhysRevE.75.011801.

URL https://link.aps.org/doi/10.1103/PhysRevE.75.011801

[5] J. J. Crassous, A. M. Mihut, L. K. Månsson, P. Schurtenberger, Anisotropic responsive microgels with tuneable shape and interactions, Nanoscale 7 (2015) 15971-15982. doi:10.1039/C5NR03827H.

URL http://dx.doi.org/10.1039/C5NR03827H

[6] L. A. Lyon, A. Fernandez-Nieves, The polymer/colloid duality of microgel suspensions, Annual Review of Physical Chemistry 63 (1) (2012) 25-43. doi:10.1146/annurev-physchem-032511-143735.

URL https://doi.org/10.1146/annurev-physchem-032511-143735

[7] F. Rodríguez-Díaz, A. Castellanos-Suárez, A. Lozsán, A phenomenological order approach to the volume phase transition in microgel particles, Phys. Chem. Chem. Phys. 19 (2017) 16541-16554. doi:10.1039/C7CP02567J.

URL http://dx.doi.org/10.1039/C7CP02567 J

[8] M. Bergman, N. Gnan, M. Obliols-Rabasa, J.-M. Meijer, L. Rovigatti, E. Zaccarelli, P. Shurtenberger, A new look at effective interactions 
between microgel particles, Nature Communications 9 (2019) 5039. doi:10.1038/s41467-018-07332-5.

URL https : //doi.org/10.1038/s41467-018-07332-5

[9] L. Rovigatti, N. Gnan, L. Tavagnacco, A. J. Moreno, E. Zaccarelli, Numerical modelling of non-ionic microgels: an overview, Soft Matter 15 (2019) 1108-1119. doi:10.1039/C8SM02089B.

URL http://dx.doi.org/10.1039/C8SM02089B

[10] G. M. Conley, S. Nöjd, M. Braibanti, P. Schurtenberger, F. Scheffold, Superresolution microscopy of the volume phase transition of pnipam microgels, Colloids and Surfaces A: Physicochemical and Engineering Aspects 499 (2016) 18 - 23. doi:https://doi.org/10.1016/j.colsurfa.2016.03.010.

URL http://www. sciencedirect.com/science/article/pii/S0927775716301388

[11] A. Aufderhorst-Roberts, D. Baker, R. J. Foster, O. Cayre, J. Mattsson, S. D. Connell, Nanoscale mechanics of microgel particles, Nanoscale 10 (2018) 16050-16061. doi:10.1039/C8NR02911C.

URL http://dx.doi.org/10.1039/C8NR02911C

[12] A. A. Karanastasis, Y. Zhang, G. S. Kenath, M. D. Lessard, J. Bewersdorf, C. K. Ullal, 3d mapping of nanoscale crosslink heterogeneities in microgels, Mater. Horiz. 5 (2018) 1130-1136. doi:10.1039/C8MH00644J. URL http://dx.doi.org/10.1039/C8MH00644J

[13] S. Matsui, Y. Nishizawa, T. Uchihashi, D. Suzuki, Monitoring thermoresponsive morphological changes in individual hydrogel microspheres, 
ACS Omega 3 (9) (2018) 10836-10842. doi:10.1021/acsomega.8b01770. URL https://doi.org/10.1021/acsomega.8b01770

[14] S.-h. Hsu, K.-C. Hung, Y.-Y. Lin, C.-H. Su, H.-Y. Yeh, U.-S. Jeng, C.-Y. Lu, S. A. Dai, W.-E. Fu, J.-C. Lin, Water-based synthesis and processing of novel biodegradable elastomers for medical applications, J. Mater. Chem. B 2 (2014) 5083-5092. doi:10.1039/C4TB00572D.

URL http://dx.doi.org/10.1039/C4TB00572D

[15] S. L. Cooper, J. Guan (Eds.), Advances in Polyurethane Biomaterials, Woodhead Publishing, 2016.

[16] P. Grossen, D. Witzigmann, S. Sieber, J. Huwyler, Peg-pcl-based nanomedicines: A biodegradable drug delivery system and its application, Journal of Controlled Release 260 (2017) 46 - 60. doi:https://doi.org/10.1016/j.jconrel.2017.05.028.

URL http://www.sciencedirect.com/science/article/pii/S0168365917306004

[17] L. P. Fonseca, R. B. Trinca, M. I. Felisberti, Amphiphilic polyurethane hydrogels as smart carriers for acidic hydrophobic drugs, International Journal of Pharmaceutics 546 (1) (2018) 106 - 114. doi:https://doi.org/10.1016/j.ijpharm.2018.05.034.

URL http://www.sciencedirect.com/science/article/pii/S0378517318303429

[18] Y.-P. Chen, S.-h. Hsu, Preparation and characterization of novel waterbased biodegradable polyurethane nanoparticles encapsulating superparamagnetic iron oxide and hydrophobic drugs, J. Mater. Chem. B 2 
(2014) 3391-3401. doi:10.1039/C4TB00069B.

URL http://dx.doi.org/10.1039/C4TB00069B

[19] R. Liu, Q. Zhang, Q. Zhou, P. Zhang, H. Dai, Nondegradable magnetic poly (carbonate urethane) microspheres with good shape memory as a proposed material for vascular embolization, Journal of the Mechanical Behavior of Biomedical Materials 82 (2018) 9 - 17. doi:https://doi.org/10.1016/j.jmbbm.2018.02.010.

URL http://www.sciencedirect.com/science/article/pii/S1751616118301036

[20] A. C. Weems, W. Li, D. J. Maitland, L. M. Calle, Polyurethane microparticles for stimuli response and reduced oxidative degradation in highly porous shape memory polymers, ACS Applied Materials \& Interfaces 10 (39) (2018) 32998-33009, pMID: 30184426. doi:10.1021/acsami.8b11082.

URL https://doi.org/10.1021/acsami.8b11082

[21] P. J. Driest, D. J. Dijkstra, D. Stamatialis, D. W. Grijpma, The trimerization of isocyanate-functionalized prepolymers: An effective method for synthesizing well-defined polymer networks, Macromolecular Rapid Communications 40 (9) (2019) 1800867. doi:10.1002/marc.201800867. URL https://onlinelibrary.wiley.com/doi/abs/10.1002/marc. 201800867

[22] P. J. Driest, I. E. Allijn, D. J. Dijkstra, D. Stamatialis, D. W. Grijpma, Poly(ethylene glycol)-based poly(urethane isocyanurate) hydrogels for contact lens applications, Polymer International 69 (2) (2020) 131-139. arXiv:https://onlinelibrary.wiley.com/doi/pdf/10.1002/pi.5938, 
doi:10.1002/pi.5938.

URL https://onlinelibrary.wiley.com/doi/abs/10.1002/pi.5938

[23] Z. K. Zander, G. Hua, C. G. Wiener, B. D. Vogt, M. L. Becker, Control of mesh size and modulus by kinetically dependent crosslinking in hydrogels, Advanced Materials 27 (40) (2015) 6283-6288. doi:10.1002/adma.201501822.

URL https://onlinelibrary.wiley.com/doi/abs/10.1002/adma. 201501822

[24] K. Vats, G. Marsh, K. Harding, I. Zampetakis, R. E. Waugh, D. S. W. Benoit, Nanoscale physicochemical properties of chain- and step-growth polymerized peg hydrogels affect cell-material interactions, Journal of Biomedical Materials Research Part A 105 (4) (2017) 1112-1122. doi:10.1002/jbm.a.36007.

URL https://onlinelibrary.wiley.com/doi/abs/10.1002/jbm.a. 36007

[25] P. D. Yeh, A. Alexeev, Mesoscale modelling of environmentally responsive hydrogels: emerging applications, Chem. Commun. 51 (2015) 10083-10095. doi:10.1039/C5CC01027F.

URL http://dx.doi.org/10.1039/C5CC01027F

[26] A. Ghavami, H. Kobayashi, R. G. Winkler, Internal dynamics of microgels: A mesoscale hydrodynamic simulation study, The Journal of Chemical Physics 145 (24) (2016) 244902. doi:10.1063/1.4972893.

URL https://doi.org/10.1063/1.4972893

[27] N. Gnan, L. Rovigatti, M. Bergman, E. Zaccarelli, In silico synthesis of microgel particles, Macromolecules 50 (21) (2017) 8777-8786. 
doi:10.1021/acs.macromol.7b01600.

URL https://doi.org/10.1021/acs .macromol.7b01600

[28] F. Camerin, N. Gnan, L. Rovigatti, E. Zaccarelli, Modelling realistic microgels in an explicit solvent, Scientific Reports 8 (1) (2018) 14426. doi:10.1038/s41598-018-32642-5.

URL https://doi.org/10.1038/s41598-018-32642-51

[29] S. Nikolov, A. Fernandez-Nieves, A. Alexeev, Mesoscale modeling of microgel mechanics and kinetics through the swelling transition, Applied Mathematics and Mechanics (English Edition) 39 (1) (2018) 47-62. doi:10.1007/s10483-018-2259-6.

URL https://doi.org/10.1007/s10483-018-2259-6

[30] A. J. Moreno, F. Lo Verso, Computational investigation of microgels: synthesis and effect of the microstructure on the deswelling behavior, Soft Matter 14 (2018) 7083-7096. doi:10.1039/C8SM01407H.

URL http://dx.doi.org/10.1039/C8SM01407H

[31] P. J. Hoogerbrugge, J. M. V. A. Koelman, Simulating microscopic hydrodynamic phenomena with dissipative particle dynamics, Europhysics Letters (EPL) 19 (3) (1992) 155-160. doi:10.1209/0295-5075/19/3/001. URL https://doi.org/10.1209/0295-5075/19/3/001

[32] P. Español, P. Warren, Statistical mechanics of dissipative particle dynamics, Europhysics Letters (EPL) 30 (4) (1995) 191-196. doi:10.1209/0295-5075/30/4/001.

URL https://doi.org/10.1209/0295-5075/30/4/F001 
[33] R. D. Groot, P. B. Warren, Dissipative particle dynamics: Bridging the gap between atomistic and mesoscopic simulation, The Journal of Chemical Physics 107 (11) (1997) 4423-4435. doi:10.1063/1.474784.

URL https://doi.org/10.1063/1.474784

[34] P. Español, P. B. Warren, Perspective: Dissipative particle dynamics, The Journal of Chemical Physics 146 (15) (2017) 150901. doi:10.1063/1.4979514.

URL https://doi.org/10.1063/1.4979514

[35] P. J. Flory, J. Rehner, Statistical mechanics of cross-linked polymer networks ii. swelling, The Journal of Chemical Physics 11 (11) (1943) 521-526. doi:10.1063/1.1723792.

URL https://doi.org/10.1063/1.1723792

[36] A. Mourran, Y. Wu, R. A. Gumerov, A. A. Rudov, I. I. Potemkin, A. Pich, M. Möller, When colloidal particles become polymer coils, Langmuir 32 (3) (2016) 723-730, pMID: 26717422. doi:10.1021/acs.langmuir.5b03931.

URL https://doi.org/10.1021/acs.langmuir.5b03931

[37] A. M. Rumyantsev, R. A. Gumerov, I. I. Potemkin, A polymer microgel at a liquid-liquid interface: theory vs. computer simulations, Soft Matter 12 (2016) 6799-6811. doi:10.1039/C6SM01231K.

URL http://dx.doi.org/10.1039/C6SM01231K

[38] V. Y. Rudyak, A. A. Gavrilov, E. Y. Kozhunova, A. V. Chertovich, Shell-corona microgels from double interpenetrating networks, Soft 
Matter 14 (2018) 2777-2781. doi:10.1039/C8SM00170G.

URL http://dx.doi.org/10.1039/C8SM00170G

[39] J. H. Hildebrand, R. L. Scott, The solubility of Nonelectrolytes, Reinhold Publishing Group, New York, 1950.

[40] E. Mayoral, A. G. Goicochea, Modeling the temperature dependent interfacial tension between organic solvents and water using dissipative particle dynamics, The Journal of Chemical Physics 138 (9) (2013) 094703. doi:10.1063/1.4793742.

URL https://doi.org/10.1063/1.4793742

[41] V. Lenzi, P. J. Driest, D. J. Dijkstra, M. M. D. Ramos, L. S. A. Marques, Gaff-ic: realistic viscosities for isocyanate molecules with a gaff-based force field, Molecular Simulation 45 (3) (2019) 207-214. doi:10.1080/08927022.2018.1554902.

URL https://doi.org/10.1080/08927022.2018.1554902

[42] J. Wang, R. M. Wolf, J. W. Caldwell, P. A. Kollman, D. A. Case, Development and testing of a general amber force field, Journal of Computational Chemistry 25 (9) (2004) 1157-1174. doi:10.1002/jcc.20035. URL http://dx.doi.org/10.1002/jcc.20035

[43] W. Pan, D. A. Fedosov, G. E. Karniadakis, B. Caswell, Hydrodynamic interactions for single dissipative-particle-dynamics particles and their clusters and filaments, Phys. Rev. E 78 (2008) 046706. doi:10.1103/PhysRevE.78.046706.

URL https://link.aps.org/doi/10.1103/PhysRevE.78.046706 
[44] H. Masoud, A. Alexeev, Permeability and diffusion through mechanically deformed random polymer networks, Macromolecules 43 (23) (2010) 10117-10122. arXiv:https://doi.org/10.1021/ma102052m, doi:10.1021/ma102052m.

URL https://doi.org/10.1021/ma102052m

[45] T. Sirk, Y. Sliozberg, J. Brennan, M. Lisal, J. Andzelm, An enhanced entangled polymer model for dissipative particle dynamics, J. Chem. Phys. 136 (2012) 134903.

[46] S. Plimpton, Fast parallel algorithms for short-range molecular dynamics, Journal of Computational Physics 117 (1) (1995) 1 - 19. doi:10.1006/jcph.1995.1039.

URL http://www.sciencedirect.com/science/article/pii/S002199918571039X

[47] G. S. Grest, K. Kremer, Statistical properties of random crosslinked rubbers, Macromolecules 23 (23) (1990) 4994-5000. arXiv:https://doi.org/10.1021/ma00225a020, doi:10.1021/ma00225a020.

URL https://doi.org/10.1021/ma00225a020

[48] G. Kacar, E. A. J. F. Peters, G. de With, Mesoscopic simulations for the molecular and network structure of a thermoset polymer, Soft Matter 9 (2013) 5785-5793. doi:10.1039/C3SM50304F.

URL http://dx.doi.org/10.1039/C3SM50304F

[49] R. Everaers, S. K. Sukumaran, G. S. Grest, C. Svaneborg, A. Sivasubramanian, K. Kremer, Rheology and microscopic topology of entangled polymeric liquids, Science 303 (5659) (2004) 823-826. doi:10.1126/sci- 
ence.1091215.

URL https://science.sciencemag.org/content/303/5659/823

[50] M. Kröger, Shortest multiple disconnected path for the analysis of entanglements in two- and three-dimensional polymeric systems, Computer Physics Communications 168 (3) (2005) 209 - 232. doi:https://doi.org/10.1016/j.cpc.2005.01.020.

URL http://www.sciencedirect.com/science/article/pii/S0010465505002808

[51] S. Shanbhag, M. Kröger, Primitive path networks generated by annealing and geometrical methods: insights into differences, Macromolecules 40 (8) (2007) 2897-2903. doi:10.1021/ma062457k.

URL https://doi.org/10.1021/ma062457k

[52] N. C. Karayiannis, M. Kröger, Combined molecular algorithms for the generation, equilibration and topological analysis of entangled polymers: Methodology and performance, International Journal of Molecular Sciences 10 (11) (2009) 5054-5089. doi:10.3390/ijms10115054.

URL https://www.mdpi .com/1422-0067/10/11/5054

[53] R. S. Hoy, K. Foteinopoulou, M. Kröger, Topological analysis of polymeric melts: Chain-length effects and fast-converging estimators for entanglement length, Phys. Rev. E 80 (2009) 031803. doi:10.1103/PhysRevE.80.031803.

URL https://link.aps.org/doi/10.1103/PhysRevE. 80.031803

[54] R. Auhl, R. Everaers, G. S. Grest, K. Kremer, S. J. Plimpton, Equilibration of long chain polymer melts in computer simulations, The Journal of 
Chemical Physics 119 (24) (2003) 12718-12728. doi:10.1063/1.1628670. URL https://doi.org/10.1063/1.1628670

[55] G. Kacar, Dissipative particle dynamics simulation parameters and interactions of a hydrogel, Journal of the Turkish Chemical Society Section A: Chemistry 5 (2017) 19 - 28. doi:10.18596/jotcsa.309646.

URL http://dx.doi .org/10.18596/jotcsa. 309646

[56] G. Kacar, P. T. M. Albers, A. C. C. Esteves, G. de With, Mesoscopic structure and swelling properties of crosslinked polyethylene glycol in water, Journal of Coatings Technology and Research 15 (4) (2018) 691-701. doi:10.1007/s11998-018-0065-4.

URL https://doi.org/10.1007/s11998-018-0065-4

[57] V. Lenzi, P. J. Driest, D. J. Dijkstra, M. M. Ramos, L. S. Marques, Investigation on the intermolecular interactions in aliphatic isocyanurate liquids: revealing the importance of dispersion, Journal of Molecular Liquids 280 (2019) 25 - $33 . \quad$ doi:https://doi.org/10.1016/j.molliq.2019.01.165.

URL http://www.sciencedirect.com/science/article/pii/S0167732218359555 